\title{
Team Knowledge Networks, Task Dependencies and Coordination Preliminary Findings from Software Teams
}

\author{
J. Alberto Espinosa \\ Kogod School of Business \\ American University \\ alberto@american.edu
}

\author{
Mark A. Clark \\ Kogod School of Business \\ American University \\ mark.clark@american.edu
}

\author{
Emma Nordbäck \\ Hanken School of Economics \\ emma.nordback@hanken.fi
}

\begin{abstract}
Today's work increasingly involves teams with fluid boundaries, and members working on multiple projects at a time. To understand how work is effectively coordinated in such complex organizations, we focus on the role of a company's task dependency network. We integrate three research streams coordination, team knowledge and social networks to conceptualize multiteam work as a large collaboration with members in multiple functional roles and areas of expertise, with complex task dependency relationships, operating as a coherent and well-coordinated knowledge network. Through this integration and empirical test of associated hypotheses with data from a European software company, our study illustrates how to represent multiple relationships in one complex multiplex network. This extends our understanding of how the various knowledge relationships and individual attribute differences influence the effective coordination in collaborative software development work. We address the concepts of awareness and shared familiarity and how they affect coordination, while keeping our focus on illustrating the power of network analytics to gain nuanced insights into the drivers of effective coordination.
\end{abstract}

\section{Introduction}

The work of modern organizations requires coordination, defined as "the management of dependencies among task activities," [1], among its members. Coordination challenges increase rapidly as tasks become more complex, which may be particularly true in technical tasks, and as teams become larger [2]. We argue that coordination, although more often measured within formal teams existing in the organizational chart, has a structure based on the dyadic dependency relationships between team members, and that these pair-wise dependencies, collectively form a collaboration network composed of actors and relationships between these actors. How such network relationships influence collaboration has received little attention in research, even though we have known about their importance for decades [3].
We argue that delineating how members of such a network share knowledge, communicate and otherwise relate to each other are key in understanding coordination of large, collaborative, complex tasks.

In this study, we investigate the relationship between task dependencies, shared task familiarity and coordination. We use survey data collected from software teams in a software organization in Northern Europe. This data is of three types: individual (i.e., attributes of participants), dyadic (i.e., pair-wise relationships) and bipartite (participant data across various knowledge categories). We illustrate how this complex variety of data formats can be used effectively to depict how members operate as one multiplex network containing multiple relationships, which allow us to gain significant statistical power and offer insights into the factors that lead to effective collaboration in software development.

Various theoretical lenses have been applied to the understanding of collaborative work, especially recently with the increased amount of virtual work and telework. We are particularly interested in three such theoretical perspective that have received a lot of attention in the literature, but with little integration: (1) coordination - both from behavioral [4] and cognitive perspectives [5]; (2) team knowledge - there are numerous constructs, labels and theories about it in the extant literature [6], generally categorized by knowledge types, including shared schemas, fleeting knowledge [7] and familiarity [8]; and (3) social networks - analyzing dyadic relationships between multiple network actors [9]. While we have learned much about these three areas, there has been little empirical or conceptual research integrating their perspectives. This may be a missed opportunity because coordination and team knowledge are inherently social constructs best understood as relations in a network [10]. Our study contributes by addressing this gap in the literature, providing interesting directions for future research on collaborative technical and knowledge work.

In the next section of the paper we articulate our theoretical foundations and offer hypotheses. These hypotheses offer insights that extend theory about how members interact in complex organizations, identifying interesting predictions about coordination effectiveness 
that illustrate the increased explanatory power of the network analytic approach, discussed in the next section. We then discuss our methods, including data collection, variables and models. We then present our results and offer some concluding remarks and limitations.

\section{Theoretical Foundations and Study Hypotheses.}

\subsection{Coordination in Collaborative Work}

Of the few streams of research on coordination, two of them are of particular interest for this study. The organizational research literature has traditionally viewed coordination from a behavioral lens, classifying coordination as either "mechanistic" (achieved by programming the most routine aspects of the task) or "organic" (achieved by communication or feedback) for more unpredictable aspects of the task [11, 12]. In contrast, the team cognition literature has viewed coordination as an implicit mechanism where members manage their task dependencies through unspoken assumptions about what others are likely to do, based on their knowledge of the task and team $[13,14]$. There are abundant team cognition constructs in the literature [6], many of which provide arguments or empirical evidence for their positive effect on coordination, including shared mental models [15], team mental models [16], group situational awareness [17], transactive memory [18] and familiarity [8, 19], among others. However, most empirical studies from these perspectives, view coordination as an aggregate activity of the collective. In our study, we take a network analytic approach and focus on general communication, as key behavioral coordination mechanism, and on awareness and familiarity as key cognitive coordination mechanisms. We argue that it is most effective to focus on collaborators that have dependencies with each other because members that can work independently will not benefit as much from coordination.

\subsection{Team Knowledge: Awareness and Familiarity}

Situational awareness is fleeting knowledge, which is only relevant during specific situations [20,21]. When these situations pass, the particular awareness knowledge is no longer relevant. Such fleeting knowledge can be about many things, but the most relevant types for working teams is task awareness and team awareness. Awareness is defined as up-to-the minute knowledge of the activities of others and how they affect one's own activities [22]. Team awareness may involve knowing who is around or who has done what. Task awareness is about knowing how the task itself has progressed at a given point in time [23-26]. Team members need to have individual awareness of task situations to carry out their task responsibilities, but they also need to have team awareness to work together as a coordinated unit [27].

Familiarity is "the knowledge that members of a team have about the unique aspects of their work" [28], typically construed as held at the individual level, such as knowledge about the task itself and about other members on the team [29]. Familiarity differs from awareness in that it is not fleeting or situational, but long-lasting and, therefore, relevant throughout the task duration. As members of a team work together over time, they become familiar with various aspects of the task and with each other [30], and develop a common knowledge base through which team interaction and location of expert sources in the team can occur [31]. Studies have shown the positive benefits of familiarity on team performance in mining [19], flight simulation [32], problem solving [29, 33], software development [8] and various other tasks [34]. While individual familiarity is indeed important for individual performance, our interest is the shared portion of familiarity, which we argue is important for successful coordination of group tasks.

\subsection{Knowledge Networks}

While social networks and social network analysis have been around since the 1930s, more recent research has begun to employ social network theories and methods to understand knowledge networks. The thrust of these arguments is that team knowledge is inherently a social construct in which individuals share and exchange knowledge through communication and actions, creating cognitive relationships and structures that help explain team processes, coordination and performance [10]. The coordination and team knowledge research streams we discussed above have made it very clear that behavioral and cognitive coordination are important for team performance. We argue that a network analytic approach is ideally suited to provide such insights.

The team cognition literature contains abundant empirical evidence that team knowledge of many shapes and forms contribute to coordination and performance. But the majority of studies have tested these effects only with simple dyads or aggregate measures in small teams [10]. More recent research suggests that collective knowledge is not always best represented by an aggregate construct, but rather, there are deep and complex knowledge relationships among team members, resulting in team knowledge network 
structures that have a strong influence on how the team coordinates and performs as a whole. This notion of viewing team knowledge as a network has been incorporated into studies of transactive memory and knowledge collaboration [35], scientific knowledge sourcing learning [36], knowledge amplification in product development [37], collaboration in biotechnology [38] and knowledge transfer and innovation [39], among others. A recent study provided more specific arguments, concepts and methods for how to investigate team knowledge from a network perspective, giving empirical evidence of a significant increase in predictive power when network structure variables are incorporated into the analysis [10]. These methods can be applied to the question of whether specific shared processes of familiarity and communication support team coordination and performance.

\subsection{Study Hypotheses}

Our study hypotheses are modeled in Figure 1. As discussed earlier, prior research provides encouraging evidence of potential benefits of using a network analytic approach to studying the effect of team knowledge on coordination and performance [10]. While this may not be as apparent with smaller teams working in fast-paced real-time tasks, we argue that, as teams become larger, and as the task becomes more complex, asynchronous and longer in duration, the structural properties of team knowledge networks provide more explanatory power to understand what drives coordination and performance.

A dependency in a task is the "extent to which personnel are dependent on one another to perform their individual jobs" [40]. Because the management of task activity dependencies is the essence of coordination [1] we argue that the task dependency networks is a key starting point in our theoretical development. Two members responsible for task activities that are relatively independent are less likely to benefit from cognitive or behavioral coordination [41]. However, the coordination demands on members with substantial dependencies will be larger. Thus, we posit that:

H1: Task dependencies between members has a negative effect on coordination effectiveness - i.e., it increases the time and effort need by members.

We further argue that members who rely on each other for leadership will have a more coordinated collaboration. While leadership is "a process whereby intentional influence is exerted over other people to guide, structure and facilitate activities and relationships in a group or organization" [42, p.2], leadership in large collaborations is often shared and it needs to be coordinated to be effective [43]. When leadership is shared, members are more willing to accept each other's influence [44] and, consequently, have more opportunities to interact, rely on each other, and build trust [45]. This distribution of control is likely to create an environment where team members more frequently and intentionally coordinate their efforts. Thus, we anticipate dyads with a leadership relationship to be more effectively coordinated.

\section{H2: Dyads with a stronger leadership} relationship will be more effectively coordinated.

Situational awareness is a form of fleeting knowledge that enables members to notice changes in the task environment and understand the implications of these changes for the task at hand [7]. Group situation awareness research suggests that awareness of the task and about each other makes the collaboration more effectively coordinated [17], but there is little empirical evidence of this. Thus, we posit:

H3: Members of dyads with more awareness of each other will be more effectively coordinated.

Team communication is a form of behavioral coordination (by feedback), which is theorized to help collaborators coordinate effectively [40]. While we hypothesize a positive relation between team communication and effective coordination, we do this with caution because communication is theorized to be effective when the task at hand is non-routine, but somewhat inefficient and even wasteful if the task at hand is routine, so the evidence is inconclusive [40].

H4: Communication frequency between collaborators has a positive effect on coordination effectiveness.

As we discussed above, task and team familiarity have been found to have positive effects on team outcomes in various types of tasks [34], including software development [8], but there is insufficient evidence about these effects when familiarity is shared. We argue that when familiarity is shared between collaborators, it makes task coordination more effective. Thus, we posit:

H5: Team familiarity - i.e., familiarity with a colleague's knowledge, skills and interaction stylehas a positive effect on coordination effectiveness.

H6: Shared task familiarity - i.e., familiarity with a colleague's task has a positive effect on coordination effectiveness.

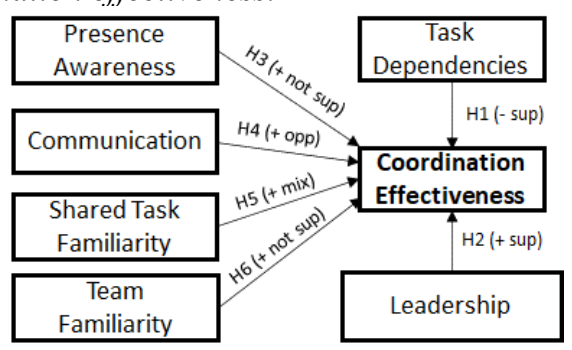

Figure 1: Study Hypotheses 


\section{Method}

\subsection{Study Sample}

The sample for our study consists of members of an IT department employing 170 workers from a software company in Northern Europe. We received 155 responses from these employees to both survey waves ( $80 \%$ response rate), but some of these responses had missing data for some variables, so we ended up with 131 usable responses with complete data.

\subsection{Data Types}

Prior to the survey, we had one-to-one meetings with six employees from different functional roles to better understand the knowledge domains to use in our survey. We then administered two surveys waves, a few weeks apart. In the first wave we gathered member attribute data (i.e., demographics) and data about who depended on whom to complete the task, which we used to build the respective task dependency network (see below), gathering three types of data, with all responses in a 1-7 Likert scale:

- Member attribute data (first wave) - containing one observation for each participant with demographic and other relevant control data like age group (from 1 - under 20 to 7 - over $60 \mathrm{yrs}$ ); number of years in the company; number of years of experience in the present functional role; gender; nationality and functional role.

- Relational data (both waves) - we collected relational data in two survey waves. Because network data requires each participant to provide responses about the others, we limited such responses to those with whom the responding member had a dependency relationship (rather than asking each person to rate 130 others on each relational question). Thus, in the first wave, we gathered data about member Task Dependencies by asking who they depended on to get their work done, with adapted survey items from prior research [41]. This yielded a directed dependency network with 611 dyads, creating a more meaningful picture of how work is accomplished and amplifying the statistical power of our analysis. In the second survey wave, we asked relationship questions only for dyads with task dependencies, consistent with coordination being about the management of such dependencies.

We measured the Perceived Coordination Delay between the two dyad members as member 1's perception of coordination delay with member 2 . We then reversed these values so that low values of coordination delay translate into high values of Perceived Coordination Effectiveness.
Besides task dependencies, we asked question about whether each dyad had a leadership relationship (i.e. how much member 1 relied on member 2 for leadership, adapted from [43]), whether they had awareness of the other member's tasks and about the member's whereabouts (i.e., presence), frequency of communication with the other dyad member, familiarity with the other dyad member's knowledge and skills, and familiarity with the dyad member's interaction and communication preferences (measures adapted from $[8,41]$ ).

- Bipartite data (second wave) - also known as 2-mode network data [46]. We collected this data for all the task familiarity questions. Essentially, this data format has one observation for each participant and one column for each knowledge area of interest (2 modes: member $\mathrm{x}$ functional task areas). The participants rated their knowledge in each of these task areas. We asked questions about several relevant aspects of their software task and averaged the responses across broader categories (for comparability with the other organization): business requirements; software methods and tools; management and administrative processes, procedures and policies; service and operations; software development languages and processes; and technical infrastructure.

\subsection{Network Data Transformations}

We collected the three types of data above to be able to construct network socio-matrices for each variable of interest. A socio-matrix is a matrix that contains one row and one column for each member of the network, with each cell measuring a particular relationship value between the row member and the column member. For example, the cell in row 4 and column 6 of the task dependency socio-matrix contains a value from 1 to 7 measuring how much member 4 depends on member 6 to complete the task. Because we had three types of data, we did some traditional network data manipulations to transform all the relevant data to a socio-matrix format, as follows:

- Member attribute data - these are various control variables for our model. We took each of the individual attribute values and "matricized" them into network data, which simply means converting individual to relational data. This can be done in many ways. For our study, we matricized the data as follows: age group difference - each cell in the sociomatrix contains the difference in age groups between the row and column member; difference in years with the organization, between the row and column member; difference in years of experience in the current role - same method; gender difference - 
same gender vs. different gender; nationality and role differences - same method.

- Relational Data - participants provided responses about members in their dependency network, yielding dyadic and directed data in network-ready format, so there was no need for transformations.

- Aggregate Bipartite Data - any 2-mode sociomatrix can be converted into various 1-mode sociomatrices using a number of methods [46]. Since the bipartite matrix contained measurements in six different knowledge area, we converted this matrix into a 1mode network using the correlation method. This method involves taking the knowledge scores in each area for each pair of members and computing the member-to-member correlation across all task areas. The value in a cell of the resulting sociomatrix contains a correlation value between -1 and 1 , measuring how much the knowledge of the two members correlates. Two members with a correlation close to 1 will have very similar knowledge in all areas, that is, a strong shared task familiarity. Negative values represent negatively correlated knowledge and values close to zero represent uncorrelated knowledge between the members.

- Detailed Bipartite Data - The aggregate bipartite data we used to compute shared task familiarity was not significant in our model, so we conjectured that these aggregate correlation values were not very meaningful, because two members may have strong positive correlation on one knowledge area (e.g. business requirements), but a negative correlation on another (e.g., infrastructure). Therefore, we modeled each of the six types of task knowledge separately by computing the difference in knowledge rating between the row and column member and reversed the result to convert differences into similarity or shared familiarity. We labeled these metrics as "Similarity." However, two members who have very low knowledge values in one area will have the same similarity metric as two members that have a very high value, if the differences are similar. So, to control for the magnitude of the knowledge shared, we also computed a metric suffixed "Minimum", which contains the minimum knowledge value in the particular task area between the two members. This is a popular approach [10] because 2 members can only share knowledge up to the knowledge held by the least knowledgeable member. Anything beyond that would be unshared. Other popular methods include maximum and average values for the dyad.

\subsection{Predictive Model}

The procedure above yielded several sociomatrices, one for each relationship of interest for the study.
Because these dyadic observations are not totally independent (i.e., a member of dyad may appear in other dyads), the Ordinary Least Squares (OLS) regression assumption of data independence is violated, so we cannot use OLS. Therefore, we employed the “Quadratic Assignment Procedure" for multivariate regressions (MRQAP) [47]. This is a commonly used network analysis method, formulated to estimate regression models with sociomatrices that contain relational data [46]. The MRQAP method yields identical regression coefficients to OLS. But the resulting $\mathrm{p}$-values have a different meaning.

In a traditional OLS regression, the p-values measure whether the coefficients are significantly different than 0 (i.e., no effect). In MRQAP, the p-value measures whether the observed effect in the data is different than what one would observe with randomly generated sociomatrices, which is a concept somewhat related to bootstrapping. The procedure starts by fitting an OLS regression and computing the respective regression coefficients, which are the observed coefficients. Each sociomatrix is then shuffled randomly by shifting rows and columns, yielding one random matrix for each relationship of interest. These random matrices are then used to estimate an OLS regression and obtain random regression coefficients.

This randomized shuffling of the sociomatrices is then repeated many times. We used the netlm function in the R package statnet to fit our model, which has a default value of 2,000 random matrix permutations, which is adequate for most estimations. The resulting p-value of each coefficient does not measure statistical significance, but the proportion of times that the random coefficient was larger than the observed coefficient (or smaller if the coefficient is negative). So, for example, a $\mathrm{p}$-value $=0.03$ indicates that the random coefficient was larger than the observed coefficient in $3 \%$ of the permutations. If the random coefficients are consistently smaller than the observed coefficients, it provides confidence that there is indeed an effect.

Using Coordination Effectiveness as the dependent variable sociomatrix, we modeled all the remaining sociomatrix variables-constructed from the matricized individual attributes, collected directly as relational data, and computed from the bipartite data. Because the aggregate shared task familiarity was not significant, we converted the respective bipartite task knowledge data into six sociomatrices, one for each task knowledge areas in our data.

\section{Results}

Our results are summarized in Table 1. Some of our hypotheses were supported and some were not. Nevertheless, our network analytics approach provided 
us with increased statistical power and deeper insights. In this section, we describe our main findings and, in the next two sections we provide a deeper discussion of our results and contributions. Our results show that differences in tenure years, years of experience and nationality were not associated with coordination effectiveness. Interestingly, differences in the age group and functional role of a dyad had a moderate positive association with coordination effectiveness $(\beta$ $=0.070, p=0.065$ and $\beta=0.121, p=0.062$, respectively), suggesting that senior colleagues collaborating with junior colleagues tend to be more coordinated than seniors with seniors or juniors with juniors, and members in different functional roles are more coordinated than members in the same role. Equally interesting was that gender differences had a positive association with coordination effectiveness $(\beta$ $=0.192, \mathrm{p}=0.002$, suggesting that males collaborating with females tend to be more coordinated than males with males or females with females.

With respect to relational variables, we found support for $\mathrm{H} 1$ that increased task dependencies within a dyad have a negative association with coordination effectiveness $(\beta=-0.045, p=0.038)$. We also found support for $\mathrm{H} 2$ that dyads with a stronger leadership relationship are more effectively coordinated $(\beta=$ $0.061, \mathrm{p}<0.001)$. We found mixed support for H3. Contrary to our expectations, task awareness was unrelated to coordination effectiveness, but presence awareness had a strong positive association $(\beta=0.239$, $\mathrm{p}<0.001)$.

Also, not entirely surprising, communication frequency between dyadic members was associated with less coordination effectiveness, which was counter to H4. But, as we discussed previously, sometimes collaborators communicate too much, beyond what is immediately needed to manage non-routine dependencies, which can have a negative effect on coordination. Prior studies have found similar negative effects and conjectured that uncoordinated teams often need to communicate more to resolve their issues [41]. We also found mixed support for H5 about the effects of team familiarity. Surprisingly, team familiarity did not have a significant effect on coordination effectiveness, but team interaction familiarity $\operatorname{did}(\beta=$ $0.372, p<0.001$ ), suggesting that having familiarity with the skills and knowledge of collaborators is not that important in this task, provided that collaborators know their peer interaction and communication preferences. Since our team familiarity measure is dyadic, perhaps it is a given that the two members of the collaborating dyad already have some familiarity with each other, all other things being equal (e.g., task dependencies, leadership relationship, presence awareness, etc.). However, having familiarity about their interaction and communication preferences is beneficial.

Table 1 - MRQAP Regression Results

\begin{tabular}{|c|c|c|}
\hline Estimate & Coefficient & P-Value \\
\hline Intercept & 2.088 & 0.000 \\
\hline \multicolumn{3}{|c|}{ Control Variables, Matricized: Differences in } \\
\hline Age Group & 0.070 & 0.065 \\
\hline Tenure Years & -0.007 & 0.128 \\
\hline Years of Experience in Role & 0.001 & 0.458 \\
\hline Gender & 0.192 & 0.002 \\
\hline Nationality & -0.023 & 0.366 \\
\hline Role & 0.121 & 0.062 \\
\hline \multicolumn{3}{|l|}{ Relational Variables } \\
\hline Task Dependency & -0.045 & 0.038 \\
\hline Leadership & 0.061 & 0.000 \\
\hline Task Awareness & -0.006 & 0.421 \\
\hline Presence Awareness & 0.239 & 0.000 \\
\hline General Communication & -0.081 & 0.012 \\
\hline Team Familiarity & 0.040 & 0.252 \\
\hline Team Interaction Familiarity & 0.372 & 0.000 \\
\hline \multicolumn{3}{|c|}{ Bipartite Data Converted to 1-Mode: Shared Task Familiarity } \\
\hline Business Requirements Similarity & -0.085 & 0.062 \\
\hline Business Requirements Minimum & 0.002 & 0.479 \\
\hline Methods \& Tools Similarity & -0.008 & 0.432 \\
\hline Methods \& Tools Minimum & -0.016 & 0.371 \\
\hline Management \& Admin Similarity & 0.070 & 0.036 \\
\hline Management \& Admin Minimum & -0.030 & 0.250 \\
\hline Service \& Operations Similarity & -0.045 & 0.099 \\
\hline Service \& Operations Minimum & 0.011 & 0.379 \\
\hline SW Development Similarity & 0.005 & 0.472 \\
\hline SW Development Minimum & 0.060 & 0.182 \\
\hline Technical Infrastructure Similarity & 0.151 & 0.000 \\
\hline Technical Infrastructure Minimum & -0.123 & 0.017 \\
\hline \multicolumn{3}{|c|}{$\begin{array}{l}\text { Residual standard error: } 0.7472 \text { on } 569 \text { degrees of freedom } \\
\text { F-statistic: } 11.05 \text { on } 25 \text { and } 569 \text { DF, p-value: }<0.001\end{array}$} \\
\hline \multicolumn{3}{|c|}{ Multiple R-squared: 0.3268 Adjusted R-squared: 0.2973} \\
\hline
\end{tabular}

With respect to the bipartite task familiarity data, as we discussed previously, our aggregate metric of shared task familiarity did not have a significant effect on coordination effectiveness. We concluded that this metric was not effective for two reasons. First, it measures shared task familiarity as a correlation of the task familiarity values of the dyadic members across all task functional areas. Correlation simply measures if the proportions of task familiarity of two members have similar patterns. However, two members could have very little familiarity about almost everything and still have highly correlated task familiarity. Or it could be both very high and still yield the similar correlation values. Second, as other studies have pointed out, familiarity can be about many things and at many levels (e.g., details vs. general) [8]. So, shared familiarity at an aggregate level may not be such a useful metric. Consequently, we drilled down into the six task 
familiarity areas, which our own participants helped us identify as relevant for their work. This is precisely where the power of network analytics resides, that is, in the ability to analyze the network at very high levels of aggregation or at more detailed levels.

As we mentioned before, we constructed two measures of shared task familiarity for each of the six task functional areas. The first one is simply the difference in individual task familiarity values between the two members of the dyad but reversed (by subtracting from 7) to represent similarity rather than difference. However, as with the case of correlation, two members may have very low values of task familiarity in one area and still have the same measure of shared task familiarity of two members with very high values. So, we developed a second metric to control for this issue by taking the minimum value of the two task familiarity scores of the dyadic members. Altogether, this yielded twelve measures -2 shared task familiarity measures $\times 6$ functional task areas. Naturally, with so many metrics we obtained very nuanced results, but providing mixed support for H6 about the effect of shared task familiarity on coordination effectiveness.

Of all the functional task areas, we found a few moderately and highly significant positive and negative effects. Naturally, this mix of effect signs explains why the aggregate measure of shared task familiarity was not significant in explaining effective coordination. Failing to support H6, neither measure of shared task familiarity for methods and tools, and for software development languages and processes were significant. Similarity in task familiarity on business requirements and on service and operations had moderate effects, but counter to H6 $(\beta=-0.085, p=0.062$ and $(\beta=-0.045$, $\mathrm{p}=0.099$, respectively), whereas the minimum task familiarity did not have a significant effect in either case. Consistent with H6, similarity in task familiarity about management and administration was positive and significant $(\beta=0.070, p<0.036)$, but the minimum value was not. Interestingly, similarity in task familiarity about technical infrastructure had a positive effect $(\beta=0.151, \mathrm{p}<0.001)$, but the minimum value had a negative and significant effect $(\beta=-0.123, \mathrm{p}=$ 0.017).

\section{Discussion}

Our goal with this analysis advances the notion of how members of complex organizations interact through a dependency structure, depicting the power of the network analytic approach to study how various forms of team knowledge influence coordination. Our results provide empirical evidence of some interesting effects. With respect to individual attributes, we found that differences in age groups, gender and functional roles improved coordination effectiveness. This supports the notion that diversity in collaboration is beneficial. Seniors coordinate better with juniors; males coordinate better with females; and technical staff coordinate better with business staff. We conjecture that software development for specific applications, like construction, require fresh diverse perspectives, and collaborators appear to devote more time and effort to coordinate with diverse peers. Interestingly, differences in time with the company and years of experience did not have a significant effect.

Our results from the relational variables are the mostly significant. Most social network studies collect and analyze this type of relational data, so this was not unexpected. Our departure point was to focus on task dependencies because members who can work independently don't need to coordinate. With 131 usable responses, there are $131 \times 130=17,030$ possible ties among the respective network actors. And, of course, it would be practically impossible to collect relational data for each member about each of their 130 peers across several dyadic relationships. By focusing just on dyads with task dependencies, identified in the first wave or our surveys, we were able to reduce the number of ties to a manageable level of 611 links, which provide sufficient statistical power. Our model confirms that, holding everything else constant, an increased level of task dependencies leads to more coordination delays. This result is consistent with coordination theory, which states that such dependencies need to be managed to be coordinated.

Our results also show that leadership relationships, presence awareness and familiarity with team member interaction and communication preferences are effective in helping us manage these task dependencies and, therefore, help coordinate more effectively. Surprisingly, task awareness and team familiarity with the knowledge and skills of others had no effect. We argue that these two variables do indeed help teams coordinate, as prescribed by situational awareness [17], transactive memory [48] and expertise coordination [49] research. But we did not observe empirical effects because our measures were too general. Rather than asking respondents if they are aware about others' tasks and familiar with other members knowledge, we could refine our survey instruments to: (1) collect more recent data on task awareness because task awareness responses are very sensitive to recall [7]; and (2) test the actual familiarity members have with their collaborators, rather than simply asking them if they are familiar with each other. Interestingly, communication frequency had a negative effect on coordination effectiveness, which is consistent with prior findings that have found that communication is useful for non- 
routine tasks, but wasteful for routine tasks, and that uncoordinated teams often have to communicate more than usual to resolve their task issues [41].

The most interesting aspect of the shared task familiarity effects, with predictors constructed from bipartite data, is consistent with prior arguments that task familiarity is like trying to identify what an elephant looks like. You can take a high-level view, but when you get into the details, your findings will change depending on which task functions you are measuring, how detailed, and which aspect of the task. For example, in a prior study of software teams, the authors argued that task familiarity could be measured with respects to software program files, larger modules, even larger subsystems or even the technical environment in which the software resides [8]. This provides some explanation for why our aggregate, correlation-based task familiarity variable was not significant, but when we drilled down into the various functional task areas, we found some significant, moderately significant and non-significant effects, both positive and negative, which provides a more nuanced understanding of how task familiarity affects coordination effectiveness.

\section{Conclusions, Contributions and Limitations}

The network analytic approach offers considerable advantages in understanding how team knowledge operates in large, complex asynchronous organizational tasks. Before we collected our survey data, we had oneon-one meetings with some organizational members to help us identify relevant task areas for the study, most of whom commented on how they needed to access expertise and resources beyond their team's boundaries in order to complete their work. This suggested that the teams involved in this study were not operating as silos, but in collaboration with other organizational members, as one large collaboration network driven by task dependencies with fluid team boundaries and multiteam memberships in organizations [50].

One of our contributions in this study is the methodology. Social network analysis has been used since the 1930s when Jacob Moreno published his seminal study of friendship ties among $4^{\text {th }}$ grade classmates in the New York Times. Social network analysis and graph theory have been used for decades to understand, not only social phenomena, but also things like event relationships, computer networks, disease spread, food item relationships, and molecular behavior, among many things. Some team cognition researchers have also applied network analytics to measure complex cognitive structures like shared mental models [51]. However, little attention has been paid to understanding how the collective knowledge of a team or group of collaborators can be measured, represented and analyzed. Our study provides some steps in that direction by conceptualizing group knowledge as a collection of dyadic knowledge relationships within a network. In our specific case, we studied constructs like task and team awareness, and task and team familiarity, but any relational knowledge construct that can be measured at the dyadic level, can be used to construct a knowledge network. Once this is done, the vast amount of network analysis methods and tools available open up widely the possibilities for investigation of the effects of collective knowledge.

The network approach is powerful, but it comes with several methodological challenges, particularly when specifying shared task familiarity and task dependency networks, which need to be addressed to help us move forward. Our present study constitutes an innovative approach to the study of teams, coordination, and knowledge, which promises to generate new insights into the drivers of team coordination, performance and related outcomes. But we acknowledge that more work is needed.

Depicting team knowledge networks this way requires elicitation of task and team knowledge data from different samples, which presents methodological challenges. Overcoming these challenges required that we employ innovative methods that, to the best of our knowledge, have not been utilized before in this line of research. For example, our participants were in various management levels (e.g., technical, team supervisor, department manager) and in different roles (e.g., developer, software architect, customer lead, functional requirements analyst, business analyst, etc.). Members also belonged to different functional groups (e.g., infrastructure, user interface, front-end applications, etc.). To complicate matters more, some members belonged to more than one team and/or worked in more than one project simultaneously. Others only worked within their functional team.

We addressed these challenges by keeping team affiliation loosely defined and capturing all the various levels and roles of each member, so that we could aggregate and slice the data in various ways (e.g., in multiple teams and projects). We first captured each member's dependency ego-network (i.e., teammates who the member depended on, or teammates that depended on the member), which we then used to construct the full task dependency network. We then asked questions about relationships with teammates in the member's ego-network. This enabled us to look at both internal dependencies within teams or external dependencies across teams, and captured things such as knowledge sharing over team boundaries. 
Our last main challenge has to do with how to construct shared task familiarity networks. As we discussed earlier, the level of granularity of the task familiarity measure (e.g., general, detailed) and the actual knowledge domain (e.g., functional task, task context) present one formidable challenge. But as we move into our next steps to analyze two additional departments from another company, we will need to compare task familiarity metrics across companies that work differently and produce different software applications. We have addressed this issue by defining general categories of functional task areas (e.g., business requirements, software development method), so that our detailed survey questions are custom tailored for each organization, but responses can be summarized across standard categories, relevant across organizations.

\section{References}

[1] T. Malone and K. Crowston, "The Interdisciplinary Study of Coordination," ACM Computing Surveys, vol. 26, pp. 87-119, 1994.

[2] F. Brooks, The Mythical Man-Month: Essays on Software Engineering, Anniversary ed. Reading, MA: A. Wesley, 1995.

[3] S. Barley, "The Alignment of Technology and Structure Through Roles and Networks," Administrative Science Quarterly, vol. 35, pp. 61-103, 1990.

[4] J. Cummings, J. A. Espinosa, and C. Pickering, "Crossing Spatial and Temporal Boundaries in Globally Distributed Projects: A Relational Model of Coordination Delay," Information Systems Research, vol. 20, pp. 420-439, September 2009.

[5] R. Rico, C. B. Gibson, M. Sanchez-Manzanares, and M. A. Clark, "Building Team Effectiveness Through Adaptation: Team Knowledge and Implicit and Explicit Coordination," Organizational Psychology Review, vol. 9, pp. 71-98, 2019.

[6] J. A. Cannon-Bowers and E. Salas, "Reflections on Shared Cognition," Journal of Organizational Behavior, vol. 22, pp. 195-202, 2001.

[7] N. J. Cooke, E. Salas, J. A. Cannon-Bowers, and R. J. Stout, "Measuring Team Knowledge," Human Factors, vol. 42, pp. 151-173, 2000.

[8] J. A. Espinosa, S. A. Slaughter, R. E. Kraut, and J. D. Herbsleb, "Familiarity, Complexity and Team Performance in Geographically Distributed Software Development," Organization Science, vol. 18, pp. 613-630, July-August 2007.

[9] S. P. Borgatti, M. G. Everett, and J. C. Johnson, Analyzing Social Networks. Los Angeles: Sage, 2013.

[10] J. A. Espinosa and M. A. Clark, "Team Knowledge Representation: A Network Perspective," Human Factors, vol. 56, pp. 333 - 348, March 2014.

[11] J. March and H. A. Simon, Organizations. New York: John Wiley and Sons, 1958.

[12] J. Thompson, Organizations in Action. New York, NY: McGraw-Hill, 1967.
[13] R. Rico, M. Sanchez-Manzanares, F. Gil, and C. B. Gibson, "Team Implicit Coordination Processes: A Team Knowledge-Based Approach," Academy of Management Review, vol. 33, pp. 163-184, 2008.

[14] r. M. Wittenbaum and G. Stasser, "Management of Information in Small Groups," in What's Social about Social Cognition?, J. L. Nye and A. M. Brower, Eds., ed Thousand Oaks, California: Sage Publications, 1996, pp. 327.

[15] J. A. Cannon-Bowers, E. Salas, and S. Converse, "Shared Mental Models in Expert Team Decision-Making," in Individual and Group Decision-Making: Current Issues, J. Castellan, Ed., ed Hillsdale, NJ: Lawrence Erlbaum Associates, Inc., 1993, pp. 221-246.

[16] R. J. Klimoski and S. Mohammed, "Team Mental Model: Construct or Metaphor," Journal of Management, vol. 20, pp. 403-437, 1994.

[17] R. Wellens, "Group Situation Awareness and Distributed Decision-Making: From Military to Civilian Applications," in Individual and Group Decision-Making: Current Issues, J. Castellan, Ed., ed Hillsdale, New Jersey: Lawrence Erlbaum Associates, Inc. Publishers, 1993, pp. 267-291.

[18] D. Wegner, "Transactive Memory: A Contemporary Analysis of the Group Mind," in Theories of Group Behavior, B. Mullen and G. Goethals, Eds., ed New York, NY: Springer-Verlag, 1986, pp. 185-205.

[19] P. S. Goodman and D. P. Leyden, "Familiarity and Group Productivity," Journal of Applied Psychology, vol. 76, pp. 578-586, 1991.

[20] M. Adams, Y. Tenney, and R. Pew, "Situation Awareness and the Cognitive Management of Complex Systems," Human Factors, vol. 37, 1995.

[21] M. Endsley, "Toward a Theory of Situation Awareness in Dynamic Systems," Human Factors, vol. 37, 1995.

[22] P. Dourish and S. Bly, "Portholes: Supporting Awareness in a Distributed Work Group," in ACM Conference on Human Factors in Computing Systems (INTERCHI). 1992.

[23] S. Greenberg, C. Gutwin, and A. Cockburn, "Using Distortion-Oriented Displays to Support Workspace Awareness," Technical report, Department of Computer Science, University of Calgary, CanadaJanuary 1996.

[24] C. Gutwin, G. Stark, and S. Greenberg, "Support for Workspace Awareness in Educational GroupWare," in ACM Conference on Computer Supported Cooperative Learning (CSCL), 1995.

[25] C. Gutwin and S. Greenberg, "The Effects of Workspace Awareness Support on the Usability of Real-Time Distributed Groupware," ACM Transactions on ComputerHuman Interaction (ToCHI), vol. 6, pp. 243-281, September 1999.

[26] C. Gutwin and S. Greenberg, "The Importance of Awareness for Team Cognition in Distributed Collaboration," in Team Cognition: Understanding the Factors that Drive Process and Performance, E. Salas and S. M. Fiore, Eds., ed Washington, D.C.: American Psychological Association, 2004, pp. 177-201, ch. 9, ISBN 1-59147-103-6.

[27] R. Wellens, "Group Situation Awareness and Distributed Decision-Making: From Military to Civilian Applications," in Individual and Group Decision-Making: Current Issues, J. Castellan, Ed., ed: LEA Publishers, 1993, p. 267. 
[28] P. S. Goodman and S. Garber, "Absenteeism and Accidents in a Dangerous Environment: Empirical Analysis of Underground Coal Mines," Journal of Applied Psychology, vol. 73, pp. 81-86, 1988.

[29] G. Littlepage, W. Robison, and K. Reddington, "Effects of Task Experience and Group Experience on Group Performance, Member Ability, and Recognition of Expertise," Organizational Behavior and Human Decision Processes, vol. 69, pp. 133-147, 1997.

[30] R. Katz, "The effects of group longevity on project communication and performance," Administrative Science Quarterly, vol. 27, pp. 81-104, 1982.

[31] M. Alavi and D. E. Leidner, "Knowledge Management and Knowledge Management Systems: Conceptual Foundations and Research Issues," MIS Quarterly, vol. 25, pp. 107-136, March 20012001.

[32] B. G. Kanki and H. C. Foushee, "Communication as Group Process Mediator of Aircrew Performance," Aviation, Space, and Environmental Medicine, vol. 60, pp. 402-410, May 1989.

[33] D. H. Gruenfeld, E. A. Mannix, K. Y. Williams, and M. A. Neale, "Group Composition and Decision Making: How Member Familiarity and Information Distribution Affect Process and Performance," Organizational Behavior and Human Decision Processes, vol. 67, pp. 1-15, 1996.

[34] D. A. Harrison, S. Mohammed, J. E. McGrath, A. T. Florey, and S. W. Vanderstoep, "Time Matters in Team Performance: Effects of Member Familiarity, Entrainment, and Task Discontinuity on Speed and Quality," Personnel Psychology, vol. 56, pp. 633-669, 2003.

[35] S. L. Jarvenpaa and A. Majchrzak, "Knowledge Collaboration Among Professionals Protecting National Security: Role of Transactive Memories in Ego-Centered Knowledge Networks," Organization Science, vol. 19, pp. 377-379, 2009.

[36] J. R. Leibiskind, A. L. Oliver, L. Zucker, and M. Brewe, "Social Networks, Learning and Flexibility: Sourcing Scientific Knowledge in New Bio-Technology Firms," Organization Science, vol. 7, pp. 428-443, 1996.

[37] J. Numata and T. Taura, "A Case Study: A Network System for Knowledge Amplification in the Product Development Process," IEEE Transactions on Engineering Management, vol. 43, pp. 356-367, 1996.

[38] W. W. Powell, "Learning from Collaboration: Knowledge and Networks in the Biotechnology and Pharmaceutical Industries," California Management Review, vol. 40, pp. 228-240, 1998.

[39] W. Tsai, "Knowledge Transfer in Intraorganizational Networks: Effects of Network Position and Absorptive Capacity on Business Unit Inovation and Performance," Academy of Management Journal, vol. 44, pp. 996-1004, 2001.

[40] A. H. Van de Ven, L. A. Delbecq, and R. J. Koenig, "Determinants of Coordination Modes Within Organizations," American Sociological Review, vol. 41, pp. 322-338, April 1976.

[41] J. A. Espinosa, J. Cummings, and C. Pickering, "Time Separation, Coordination, and Performance in Technical Teams," IEEE Transactions on Engineering Management, vol. 59, pp. 91-103, February 20122012.
[42] G. A. Yukl, Leadership in Organizations. New York: Pearson, 2013.

[43] E. Nordback and J. A. Espinosa, "Effective Coordination of Shared Leadership in Global Virtual Teams," Journal of Management Information Systems, vol. 36, pp. 321-350, 2019.

[44] F. Aime, S. E. Humphrey, and J. B. Paul, "The Riddle of Heterarchy: Power Transitions in Cross-Functional Teams," Academy of Management Journal, vol. 57, pp. 327-352, 2014.

[45] J. Z. Bergman, E. E. Small, and S. Davenport, "The Shared Leadership Process in Decision-Making Teams," The Journal of Social Psychology, vol. 152, pp. 17-42, 2012.

[46] S. P. Borgatti, M. G. Everett, and L. C. Freeman, "UCINET for Windows: Software for Social Network Analysis," V ed: Analytic Technologies, 2002.

[47] S. Wasserman and K. Faust, Social Network Analysis: Methods and Applications: Cambridge University Press, 1994.

[48] K. Lewis, "Measuring Transactive Memory Systems in the Field: Scale Development and Validation," Journal of Applied Psychology, vol. 88, pp. 587-604, August 2003.

[49] S. Faraj and L. Sproull, "Coordinating Expertise in Software Development Teams," Management Science, vol. 46, pp. 1554-1568, December 2000.

[50] M. B. O'Leary, M. Mortensen, and A. W. Wooley, "Multipe Team Membership: A Theoretical Model of Its Effect on Productivity and Learning for Individuals and Tams," Academy of Management Review, vol. 36, pp. 461478, 2011.

[51] J. R. Rentsch and R. J. Klimoski, "Why do Great Minds Think Alike?: Antecedents of Team Member Schema Agreement," Journal of Organizational Behavior, vol. 22, pp. 107-120, 2001. 